ESAIM : Proceedings, Vol. 7, 1999, 349-358

Third International Workshop on Vortex

Flows and Related Numerical Methods

http://www.emath.fr/proc/Vol.7/

\title{
Flow in a 2D Duct with Moving Boundaries - Vortex Blob Approach
}

\author{
B. Protas, A. Styczek \\ Department of Aerodynamics, Institute of Aeronautics and Applied Mechanics, \\ Warsaw University of Technology, ul. Nowowiejska 24, 00-665 Warsaw, Poland \\ E-mail: bprotas@meil.pw.edu.pl
}

\begin{abstract}
In the paper we develop Random Vortex Blob method for the case of flows in periodic domains. It is shown how the formulation of the problem must be modified to account for the periodicity constraint. Properties of periodic vorticity particles are discussed. We also derive a rigorous method to satisfy the boundary conditions. Example computations concern the flow in an Xperiodic duct with moving boundaries and varying in time pressure gradient.
\end{abstract}

\section{Introduction}

In the present paper we are interested in an incompressible viscous fluid flow through an infinitely long horizontal channel. We consider two-dimensional (2D) flows and in order to properly account for the infinite length of the channel we will assume that both the flow domain and the corresponding flow quantities are X-periodic with some characteristic period $L=2 \pi$. We will investigate flows at turbulent, yet not too high, values of the Reynolds numbers. In our study the flow is driven by (i) pressure gradient (i.e. the prescribed drop of pressure across one segment of the channel) and/or (ii) the motion of the boundaries. This particular flow configuration represents phenomena taking place during digestion in living organisms as well as those encountered in process engineering. The flow configuration is outlined in Fig.1. The problem is solved using the Random Vortex Blob Method, an extension of the implementation described in [1]. However, in order to properly account for the requirement of X-periodicity, some additional problems have to be addressed. First, one must assure that the relevant hydrodynamic quantities are all X-periodic by construction. Then it will be shown that periodicity imposes an additional constraint which relates the total vorticity production in the boundary layer to the pressure drop across one segment of the duct and the acceleration of the boundaries.

The organization of the paper is as follows. In the next Section we give the mathematical description of the problem and outline the solution method, in the following Section we consider the properties of the X-periodic vorticity carrier, in Section 4 we relate the vorticity production in the boundary layer to the corresponding pressure drop, in Section 5 we present some results of the numerical simulations, and, finally in Section 6, we give some conclusions and discuss further perspectives. 


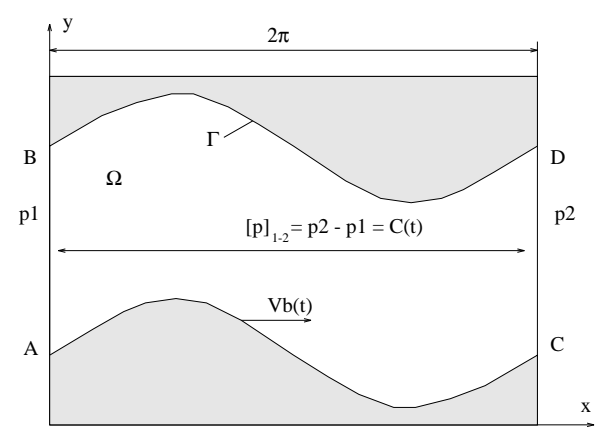

Figure 1: Flow configuration.

\section{Formulation of the Problem and the Solution Method}

The problem is described using the velocity-vorticity form of the momentum equations with appropriate initial and boundary conditions for velocity. In our investigation the flow is initially at rest and velocity boundary condition represents the prescribed motion of the duct walls. Because of the assumption of X-periodicity, corresponding constraints have to be added to the governing system

$$
\left\{\begin{array}{l}
\frac{\partial \omega}{\partial t}+(\vec{V} \cdot \nabla) \omega=\nu \triangle \omega \\
\nabla \cdot \vec{V}=0 \\
\vec{V}(x, y, 0)=0,\left.\vec{V}\right|_{\Gamma}=\vec{V}_{b} \\
\vec{V}(x, y, t)=\vec{V}(x+2 k \pi, y, t), k=0, \pm 1, \ldots \\
\nabla p(x, y, t)=\nabla p(x+2 k \pi, y, t) \\
{[p]_{1-2}=p_{2}-p_{1}=C(t) .}
\end{array}\right\}
$$

The relations (Eq.1a) represent the X-periodicity requirement for the hydrodynamic fields (note that the first of these relations implies the X-periodicity of the corresponding vorticity field $\omega=$ $\frac{\partial u}{\partial y}-\frac{\partial v}{\partial x}$ ), whereas (Eq.1b) is the varying in time pressure drop across one segment of the channel. It should be emphasized that it is strictly necessary to take this condition into account, since otherwise the problem would not be uniquely posed. This is because for given boundary velocity and the period $L$ one can obtain flows with different pressure drops.

The above system of partial differential equations (PDE's) is solved using the Random Vortex Blob Method originally introduced in [2]. Our approach is an extension of the method developed in [1], some additional measures have however to be taken in order to satisfy the periodicity constraints. In principle the method makes use of the random walk to model viscous diffusion and incorporates a rigorous algorithm which controls vorticity production in the boundary layer, so that the no-slip condition for velocity is satisfied. Below we give a brief outline of the method.

First we discretize the vorticity field on the Lagrangian grid

$$
\omega(x, y, t) \cong \sum_{i=1}^{N} \Gamma_{i} \chi_{\sigma}\left(x_{i}(t), y_{i}(t), t\right),
$$

where $\Gamma_{i}$ is the circulation of a given vortex blob and $\psi_{\sigma}$ is a function which describes the distribution of vorticity within the support of the vorticity carrier characterized by the core size $\sigma$. The 


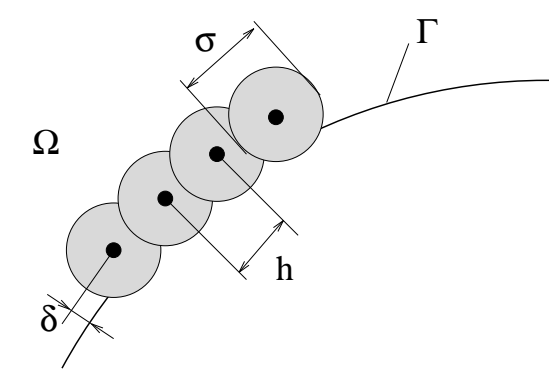

Figure 2: Locations of the new vortex blobs on the boundary.

function $\chi_{\sigma}$ must be X-periodic as it is related to an infinite array of vortices aligned along the $\mathrm{X}$-axis. In the following section we will discuss the kinematic properties of such a vortex system. Every single vortex blob may thus be regarded as a representative of an infinite family of its images. As is well known, the 2D vorticity equation is formally similar to the Planck-Fokker equation describing the evolution of the stochastic non-anticipating Wiener process [3]. Consequently, the time development of the vorticity field may be approximated as the evolution of a family of vortex blobs, each of them moving according to the stochastic Ito equation

$$
d \bar{x}=\vec{V} d t+\sqrt{2 \nu} d \bar{W}
$$

where $d \bar{x}$ is an infinitesimal displacement of a vortex carrier, $\vec{V}=[u, v]$ is the deterministic velocity field (i.e. the "drift"), $\sqrt{2 \nu}$ is the amplitude of the random walk and $d \bar{W}=\left[W_{x}, W_{y}\right]$ stand for infinitesimal increments of the Wiener process. Equation (3) can be integrated forward in time resulting in the trajectories of the vorticity carriers. In our approach the velocity field $\vec{V}$ has the representation

$$
\vec{V}=\vec{V}_{B O}+\vec{V}_{B N}+\vec{V}_{A}+\vec{V}_{\Gamma}
$$

where the constituent fields have the following interpretations:

- $\vec{V}_{B O}, \vec{V}_{B N}$ - velocity fields induced by the old (respectively $B O$ ) and new (respectively $B N$ ) generations of vortex blobs; they are a linear superposition of velocities due to particular vortices and are $\mathrm{X}$-periodic by construction (see discussion in the next section),

- $\vec{V}_{A}$ - an auxiliary potential velocity field (also periodic in the X-direction), and

- $\vec{V}_{\Gamma}$ - velocity field due to two periodic contour-connected vortices $\Gamma_{a}$ and $\Gamma_{b}$ located above and below each segment of the channel, the necessity for incorporation of these vortices will be clarified in Section 4 .

All these fields are potential everywhere, apart from $\vec{V}_{B O}$ and $\vec{V}_{B N}$ which are vortical within the supports of the vorticity carriers and are potential outside. At every time step all of the old vorticity carriers are advected and simultaneously a new generation of vortices is introduced in the immediate proximity of the wall to account for the vorticity production in the boundary layer. The locations of the new particles are schematically shown in Fig.2. The following rigorous algorithm is used to determine the circulations of the new vortices. According to (4), the tangential $V_{b}^{t}$ and 
normal $V_{b}^{n}$ components of the boundary velocity are given by

$$
\left\{\begin{array}{l}
V_{b}^{t}=V_{B O}^{t}+V_{B N}^{t}+V_{A}^{t}+V_{\Gamma}^{t} \\
V_{b}^{n}=V_{B O}^{n}+V_{B N}^{n}+V_{A}^{n}+V_{\Gamma}^{n}
\end{array}\right.
$$

We now use the Hilbert transformation [4], represented by a linear integral operator $\mathcal{L}$, which assigns the corresponding tangential component to the normal component of any given potential vector field taken on the boundary

$$
V^{t}=\mathcal{L}\left(V^{n}\right),
$$

where $V^{n}=(\vec{n} \cdot \nabla \Phi)$ and $V^{t}=(\vec{\tau} \cdot \nabla \Phi)$. Applying the above operator to the field $\vec{V}_{A}$ we obtain

$$
V_{A}^{t}=\mathcal{L}\left(-V_{B O}^{n}-V_{B N}^{n}-V_{\Gamma}^{n}-V_{b}^{n}\right)
$$

and we can thus collapse (5) into a single equation for the tangential component of the boundary velocity

$$
V_{B O}^{t}+V_{B N}^{t}+V_{\Gamma}^{t}-\mathcal{L}\left(V_{B O}^{n}+V_{B N}^{n}+V_{\Gamma}^{n}+V_{b}^{n}\right)=V_{b}^{t}
$$

This equation can be used to determine the intensities of the new vortices. It may be satisfied pointwise, at points corresponding to the projections of the vortex centers on the contour, or in the mean sense, averaged over the boundary segments which cross-sect the blobs. In our investigation we choose the latter possibility and integrate (8) over the segments $\left[s_{k}, s_{k+1}\right], k=1, \ldots, N B$, where $N B$ denotes the number of blobs in the new generation. The fields $\vec{V}_{B N}$ and $\vec{V}_{\Gamma}$ are due to vortices with fixed locations (cf. Fig.2) and therefore can have the following representations

$$
\left\{\begin{aligned}
V_{B N}^{n}(s) & =\sum_{i=1}^{N B} \Gamma_{i} N_{i}(s) \\
V_{B N}^{t}(s) & =\sum_{i=1}^{N B} \Gamma_{i} T_{i}(s) \\
V_{\Gamma_{a / b}}^{n}(s) & =\Gamma_{a} N_{a}(s)+\Gamma_{b} N_{b}(s) \\
V_{\Gamma_{a / b}}^{t}(s) & =\Gamma_{a} T_{a}(s)+\Gamma_{b} T_{b}(s)
\end{aligned}\right.
$$

where $s$ is the arc length coordinate along the contour. The functions $N_{i}(s)$ and $T_{i}(s)$ represent the normal and tangential velocity components induced by vortices with unit circulation located at $\left(x_{i}, y_{i}\right)$. The subscripts $a$ and $b$ denote contour-connected vortices located outside the flow domain, above and below the channel walls (Fig.4). Consequently, the relation (8) transforms into a system of algebraic equations

$$
K \Gamma=B
$$

where

$$
K_{i j}=\int_{s_{i}}^{s_{i+1}}\left(T_{j}-\mathcal{L} N_{j}\right)\left(s^{\prime}\right) d s^{\prime}, i=1, \ldots, N B, j=1, \ldots, N B+2
$$

are the elements of the "influence matrix" representing the mutual induction of the new vortices and

$$
B_{i}=-\int_{s_{j}}^{s_{j+1}}\left(V_{B O}^{t}+V_{B N}^{t}+V_{\Gamma}^{t}\right)\left(s^{\prime}\right) d s^{\prime}+\int_{s_{j}}^{s_{j+1}} \mathcal{L}\left(V_{B O}^{n}+V_{B N}^{n}+V_{\Gamma}^{n}+V_{b}^{n}\right)\left(s^{\prime}\right) d s^{\prime},
$$




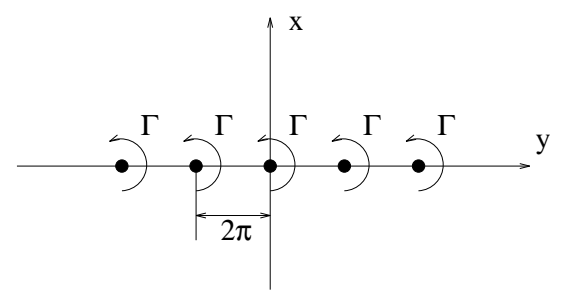

Figure 3: Construction of the X-periodic vorticity carrier.

are the right-hand side terms which stand for the perturbation of the boundary velocity due to displacement of the vorticity already present in the flow field. Consequently, the system (10) determines how the circulations of the new vortices, both those created in the boundary layer and the two which remain contour-connected, should be adjusted so as to compensate for the boundary velocity perturbation resulting from the displacement of the old blobs and acceleration of the boundary. The system therefore consists of $N B$ equations for $N B+2$ unknowns $\Gamma_{i}$ which are the circulations of the $N B$ new vortex blobs and the two contour-connected vortices $\Gamma_{a}$ and $\Gamma_{b}$. In Section 4 we will derive the two missing equations using the constraints on the vorticity production in the boundary layer.

\section{Construction of the X-periodic Vorticity Carrier}

In the present section we are concerned with the derivation of the induction formulas for the Xperiodic vorticity carrier. As was already remarked, such a vortex can in fact be represented as an infinite array of vortices aligned along the $\mathrm{X}$-axis [5]. All vortices carry the circulation $\Gamma$ and are separated by the distance $2 \pi$ from each other (Fig. 3). The velocity induced by such a system can be obtained by summing up the velocities induced by all the images (for simplicity we use the complex notation with $V(z)=(u-i v)(z)$ and $z=x+i y$, where $i$ stands for the imaginary unit)

$$
V_{\text {ind }}(z)=\sum_{n=-\infty}^{\infty} \frac{\Gamma}{2 \pi i} \frac{1}{z-\left(z_{0}+2 \pi n\right)}=\frac{\Gamma}{2 \pi i} \cot \left(\frac{z-z_{0}}{2}\right) .
$$

In the above $z_{0}$ is the location of a representative vortex $\left(z_{0}=0\right.$ for the configuration shown in Fig.3). One can easily verify that the corresponding complex potential $W(z)$ is given by

$$
W(z)=\frac{\Gamma}{4 \pi i} \ln \sin \left(\frac{z-z_{0}}{2}\right) .
$$

The real part of (14) is the Green's function of the underlying Neumann problem for the Laplace equation. Formula (13) has interesting asymptotic properties. For $y$ going to infinity the Xcomponent of the induced velocity does not decay to zero, instead it approaches some finite value $\lim _{y \rightarrow \pm \infty} V_{\text {ind }}= \pm \frac{\Gamma}{4 \pi}$. This fact however does not have any consequences for our method, since we restrict our investigation to flows in closed domains. At the origin (i.e. for $z \rightarrow z_{0}$ ) the formula (13) behaves like a single point-vortex with $V_{i n d} \stackrel{z \rightarrow z_{0}}{\longrightarrow} \frac{\Gamma}{2 \pi i} \frac{1}{z-z_{0}}$. Of course, for the purpose of desingularization, it is necessary to convolve the induction formula (13) with a suitable mollifying function. In our study we use blobs with constant vorticity distribution within the vortex core and zero outside. Consequently, formula (13) has to be modified to account for situations when $z_{0}$ lies within the core. To this end, when $\left|z-z_{0}\right|<\frac{\sigma}{2}$, we subtract from (13) the singular term $\frac{\Gamma}{2 \pi i} \frac{1}{z-z_{0}}$ 
and then add the solid body rotation represented by $\frac{\Gamma}{2 \pi i} \frac{\overline{z-z_{0}}}{\sigma^{2}}$, where the overbar denotes complex conjugation. Finally, one could observe that for an Y-periodic array of vortices the trigonometric functions in formulas (13) and (14) (i.e. cot and sin) should be replaced by their hyperbolic counterparts (i.e. coth and sinh).

\section{Solution of the Potential Problem in the X-periodic Domain}

The remaining element that has to be determined in the representation (4) is the auxiliary field $\vec{V}_{A}$. It is a potential field and therefore can be found as the gradient $\vec{V}_{A}=\nabla \Phi$ of the potential $\Phi$ which is the solution of the Neumann problem for the Laplace equation posed in the X-periodic domain

$$
\left\{\begin{array}{l}
\triangle \Phi=0 \\
(n \cdot \nabla) \Phi=V_{A}^{n} \\
\Phi(x, y)=\Phi(x+2 \pi k, y), k=0, \pm 1, \ldots
\end{array}\right.
$$

The Neumann-type boundary condition in the above problem is determined using the relation (5). This kind of the Laplace problem can be solved using a variety of methods. In our case we use the Boundary Element Method suitably modified to account for periodicity. First it is necessary to find the boundary value of the potential $\Phi$. It is the solution of the boundary integral equation

$$
\begin{aligned}
& \Phi\left(s_{P}\right)+\frac{1}{\pi} \oint_{\Gamma} \operatorname{Re}\left[\frac{1}{2} \cot \left(\frac{z-z_{0}}{2}\right) n_{Q}\right] \Phi\left(s_{Q}\right) d s_{Q} \\
& =\frac{1}{\pi} \oint_{\Gamma} \operatorname{Re}\left[\frac{1}{2} \ln \sin \left(\frac{z-z_{0}}{2}\right) n_{Q}\right] \frac{d \Phi\left(s_{Q}\right)}{d n_{Q}} d s_{Q}, P, Q \in \Gamma,
\end{aligned}
$$

where the X-periodic kernel functions have been used. The above is the Fredholm equation of the second kind and can be solved using standard techniques. At this point it is possible to reconstruct the field $V_{A}$ at any point belonging to the flow domain. To this end one can use the Cauchy-type integral with the suitable X-periodic kernel

$$
V_{A}(z)=(u-i v)(z)=\frac{1}{4 \pi i} \oint_{\Gamma} V(\tau) \cot \left(\frac{z-\tau}{2}\right) d \tau=\frac{1}{8 \pi i} \oint_{\Gamma} \frac{W(\tau)}{\sin ^{2}\left(\frac{z-\tau}{2}\right)} d \tau, z \in \Omega, \tau \in \Gamma .
$$

In these expressions $W(\tau)$ denotes the boundary value of the complex potential $W=\Phi+i \Psi$, where $\Phi$ is the solution of (16), whereas $\Psi$ (i.e. the streamfunction) can be found as $\Psi(s)=$ $\int_{0}^{s} V_{A}^{n}(\tau) d \tau+\Psi(0)$. The potential velocity $V(\tau)$ on the boundary is the derivative $\frac{d W}{d z}$ of the complex potential $W$ taken along the contour.

\section{Relation Between Vorticity Production and Pressure Drop Across One Segment of the Duct}

In the present section we derive the formula which relates vorticity production in the boundary layer to the pressure drop $[p]_{1-2}$ across one section of the channel. This relation turns out to be a necessary supplement to the governing system (1) in multiply connected domains. The review papers [6] and [7] account for the pressure constraints in the context of the velocity-vorticity 


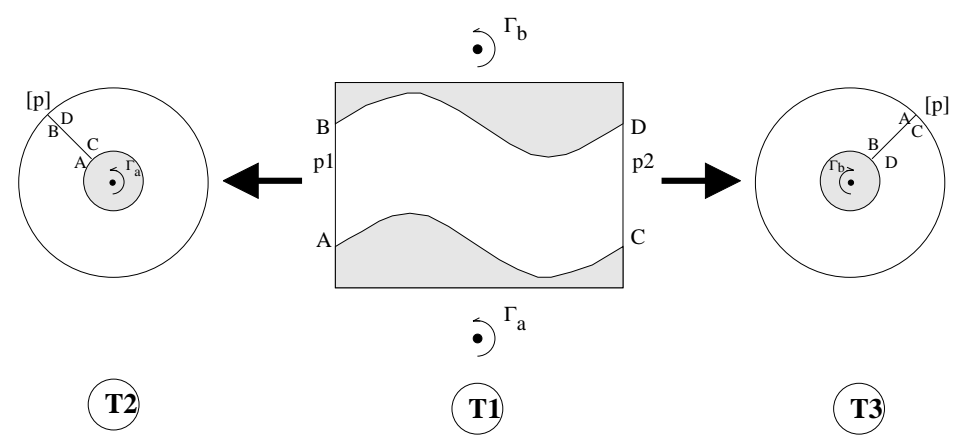

Figure 4: Topological equivalence between the flow in a periodic duct and the flow in an annular domain with a pressure jump.

formulation of the momentum equations. From the topological point of view, our flow configuration (denoted "T1" in Fig.4) is equivalent to either of the two annular configurations (denoted "T2" and "T3" in Fig.4) involving a pressure jump across the cut (i.e. between the points A-C and B-D). There is no net flow in the normal direction, so consequently there can be no pressure difference between the pairs of points A-B and C-D. We will now express the pressure jump $[p]_{1-2}$ in terms of vorticity production in the boundary layer. First, we project the Navier-Stokes equation in the Lamb form on the direction tangential to the boundary $\Gamma$

$$
\vec{\tau} \cdot \frac{\partial \vec{V}}{\partial t}-(\vec{V} \cdot \vec{n}) \omega=-\frac{\partial}{\partial s}\left(p+\frac{V^{2}}{2}\right)-\nu \frac{\partial \omega}{\partial n},
$$

where $\vec{n}$ and $\vec{\tau}$ denote versors normal and tangential to the boundary. We now integrate (18) along one segment of the channel, i.e. from A to C, or equivalently from B to D

$$
[p]_{1-2}=-\int_{A}^{C} \vec{\tau} \cdot \frac{\partial \vec{V}}{\partial t} d s+\nu \int_{A}^{C} \frac{\partial \omega}{\partial n} d s .
$$

Note that the non-linear advection term drops out due to the impermeability condition on the boundary, whereas the contribution from $\frac{V^{2}}{2}$ vanishes because of continuity of the velocity field. The above relation clearly shows that in order for the pressure drop to be equal to the prescribed value, the integral vorticity flux across the boundary must satisfy a constraint which also incorporates a term corresponding to the boundary acceleration. Equations of the type (19) can be written for each part of the boundary, in our case A-C and B-D, thus providing the two necessary equations complementing the algebraic system (10). It is now obvious that because of these integral constraints, two additional "degrees of freedom" are necessary, so that the algebraic system (10) is not overdetermined. This clarifies the role played by the two contour-connected vortices $\Gamma_{a}$ and $\Gamma_{b}$. The presented argument implies that even though we deal with an internal flow, it has some features of external flows which are due to the requirement of periodicity. In a real flow in an annular domain, or a flow exterior to a contour, the relation (19) will also hold, with the reservation however that there will be no pressure jump resulting in a single-valued pressure distribution. This implies the existence of a relation between the pressure jump and the circulation of the velocity field around the contour in all multi-connected domains. Finally, one should observe that a constraint similar to (19) naturally enters in certain formulations of the boundary conditions for the Vortex Method (e.g. [8]). 

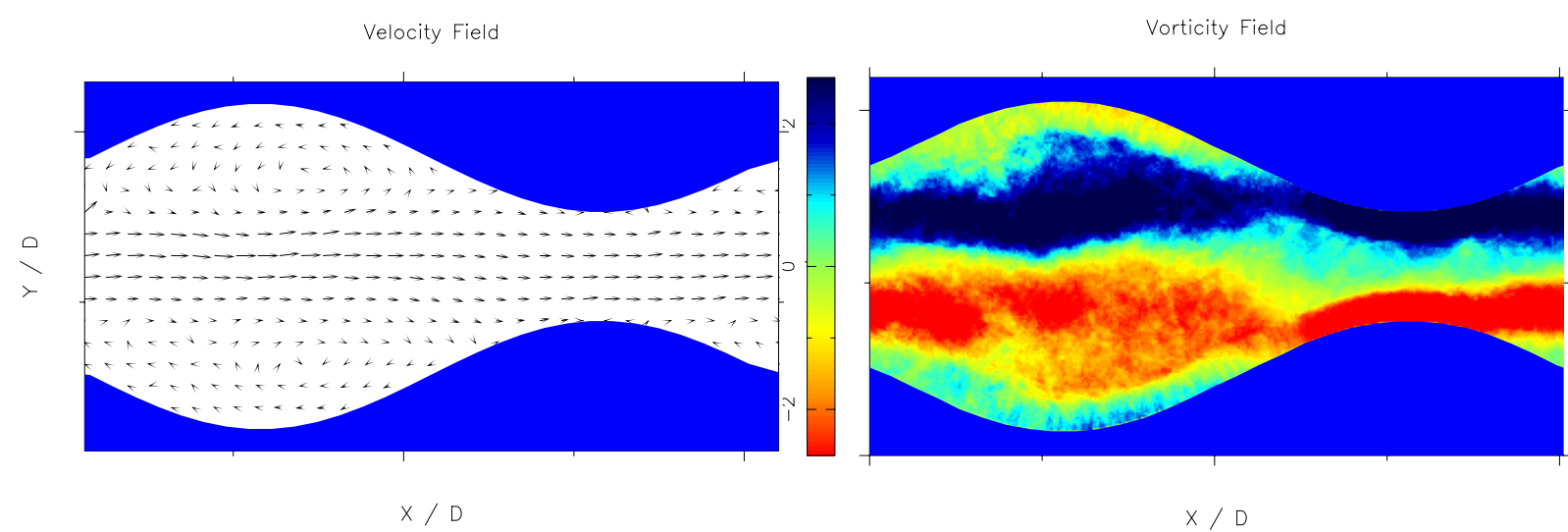

Figure 5: The velocity (left) and vorticity (right) fields in a plane $2 \pi-$ periodic channel flow. See text for details.

\section{Results of Numerical Simulations}

We now proceed to show the results of numerical computations. We will present four simulations, all of which concern the channel flow driven by variable in time pressure gradient and motion of the boundaries. The Reynolds number based on the channel height $H$, the mean flow velocity $U_{0}$ and the kinematic viscosity $\nu$ was roughly the same in all the studied configurations and close to 5000. At every time step of the simulations approximately 100 new vortices were introduced at both lower and upper boundary and the total number of vorticity particles at the end of the simulation was around $6000 \div 8000$ (note that some of the particles left the flow domain due to the random motion). Further increase of the number of vorticity carriers was prevented by prohibitive computational cost mainly related to the evaluation of vortex induction which scales as $N^{2}$. Fast summation algorithm of the type [9] for the periodic induction formula has not yet been implemented. In all our simulations computations were performed until statistically steady state was reached. In Figs.5 through 8 we show the velocity (left) and vorticity (right) fields obtained for the following four flow configurations:

- the pressure gradient is constant in time and directed to the left; the wave on the boundary moves at a constant velocity from right to left (Fig.5),

- the pressure gradient is constant in time and directed to the left; the wave on the boundary moves at a constant velocity from right to left; this case is characterized by a shorter wavelength of the bumpy disturbance on the wall (Fig.6),

- the pressure gradient is constant in time and directed to the left, the bump on the boundary oscillates horizontally with the period $T=3.75$ (Fig.7),

- the pressure gradient and the bump on the boundary oscillate horizontally with the period $T=3.75$ and opposite phases (Fig.8).

In the first three cases the net flow is from the left to the right. 
Velocity Field

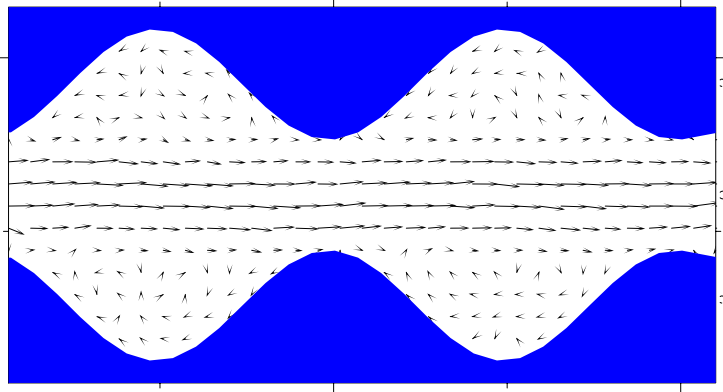

$x / D$
Vorticity Field

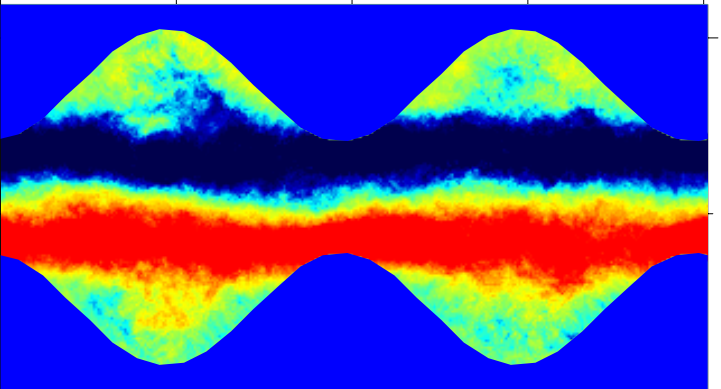

$x / D$

Figure 6: The velocity (left) and vorticity (right) fields in a plane $2 \pi-$ periodic channel flow. See text for details.

Velocity Field

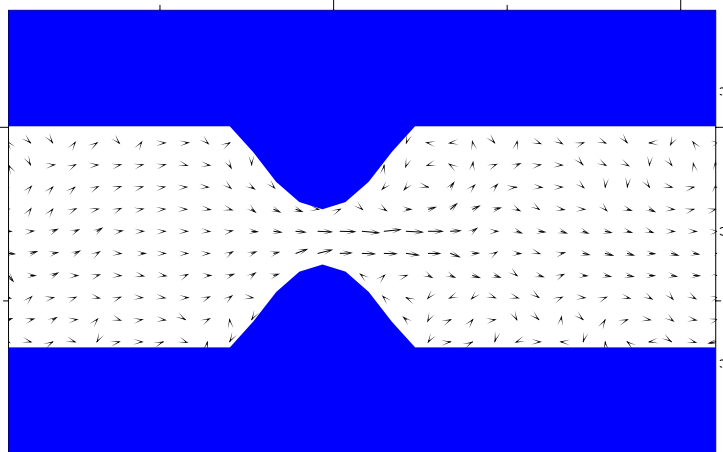

Vorticity Field

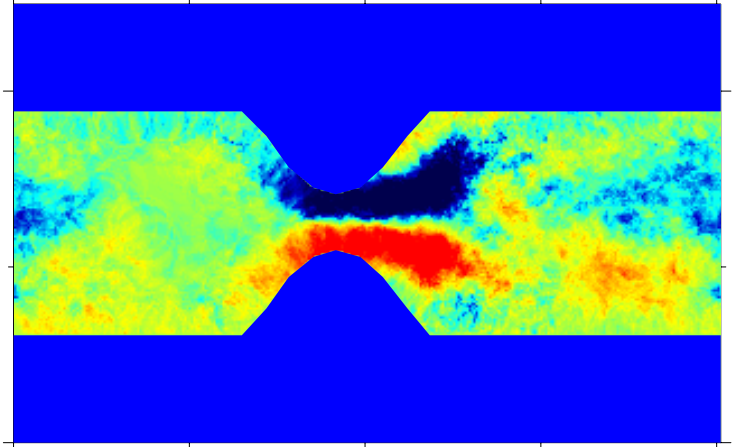

Figure 7: The velocity (left) and vorticity (right) fields in a plane $2 \pi-$ periodic channel flow. See text for details.

Velocity Field

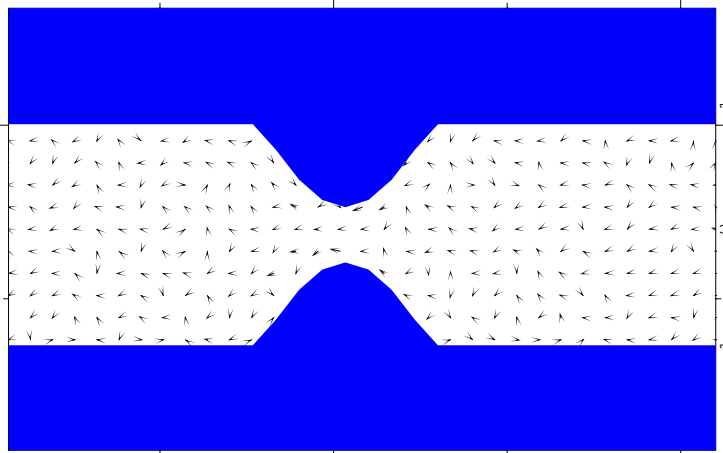

Vorticity Field

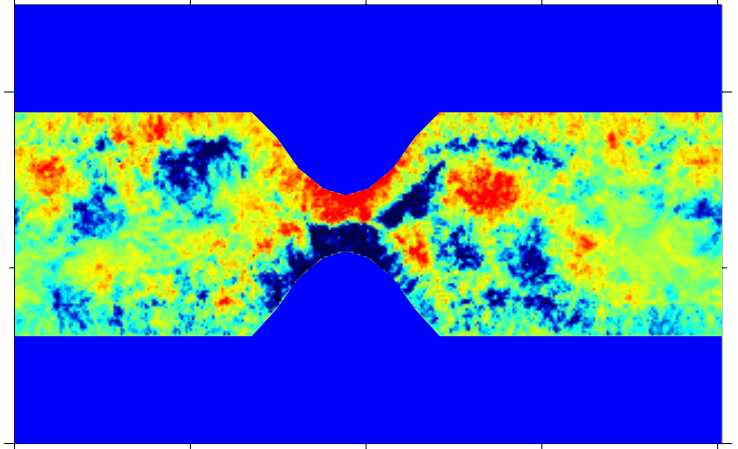

Figure 8: The velocity (left) and vorticity (right) fields in a plane $2 \pi-$ periodic channel flow. See text for details. 


\section{Conclusions and Further Perspectives}

In the present work we have extended the Vortex Blob Method for the case of flows in an X-periodic horizontal channel. It was shown that this flow configuration has some characteristics of flows in multi-connected domains. We proved that an integral constraint on the vorticity production in the boundary layer and the boundary acceleration must be supplemented to the traditional vorticity formulation of the equations of motion, so that the pressure jump across one section of the channel is equal to the prescribed value. We have furthermore developed a rigorous procedure which determines the intensities of the new vortices in the boundary layer in such a way that the "no-slip" condition for the velocity is satisfied. Particular emphasis was given to the design and properties of the X-periodic vorticity carrier. Finally, we presented some results of unsteady flow computations.

Further work will involve implementation of the fast summation algorithm for the case of X-periodic geometry. Two such methods are currently available, namely [10] and [11]. Issues related to the problem of velocity evaluation in periodic channel configurations were also addressed in [12]. Yet another improvement would consist in checking various dissipation models and the influence they may have on the accuracy of computations.

\section{Acknowledgments}

B.P. is supported by the Foundation for Polish Science. The computations were performed at the Supercomputing Center of the University of Warsaw (ICMM UW). We have benefited from enlightening discussions with J. Rokicki, J. Szumbarski and P. Wald.

\section{References}

1 Styczek, A., Wald, P.: Fast and Efficient Vortex Blob Simulation of the Flow Past the Circular Cylinder. Arch. Mech. Eng. XLII, 281 (1995).

2 Chorin, A.: Numerical study of slightly viscous flows. J. Fluid Mech. 53, 785 (1973).

3 Gardiner, C. W.: Handbook of Stochastic Methods for Physics, Chemistry and Natural Science, Springer Series in Synergetics, Springer Verlag (1985).

4 Mushalishvili, N. I.: Singular Integral Equations. Noordhaft \& Groningen, Holland (1953).

5 Saffman, P. E.: Vortex Dynamics. Cambridge University Press, Cambridge, Great Britain (1993).

6 Gresho, P. M.: Incompressible Fluid Dynamics: Some Fundamental Formulation Issues. Annu. Rev. Fluid Mech. 23, 413-453 (1991).

7 Gunzburger, M. D. Peterson, J. S.: Finite-Element Methods for the Streamfunction-Vorticity Equations: Boundary-Condition Treatments and Multiply Connected Domains. SIAM J. Sci. Stat. Comput. 9 (4), 650-668 (1988).

8 Koumoutsakos, P.: Direct Numerical Simulations of Unsteady Separated Flows Using Vortex Methods. Ph.D. Thesis, Caltech (1993).

9 Greengard, L., Rokhlin, V.: A Fast Algorithm for Particle Simulations. J. Comp. Phys. 73 (2), (1987).

10 Hamilton, J., Majda, G.: On the Rokhlin-Greengard Method with Vortex Blobs for Problms Posed in All Space or Periodic in One Direction. J. Comp. Phys. 121, (1995).

11 Sakajo, T., Окамото, H.: An application of Draghicescu's fast summation method to vortex sheet motion. J. Soc. Phys. Japan, 67 (2), (1998), pp 462-470.

12 Monaghan, J. J. and Humble, R. J.: Vortex Particle Methods for Periodic Channel Flow. J. Comp. Phys. 107, 152-159, (1993). 